\title{
3-D eigenmode calculation of metallic nano-structures
}

\author{
B. Bandlow and R. Schuhmann
}

University Paderborn, EIM-E, FG Theoretische Elektrotechnik, Warburger Straße 100, 33098 Paderborn, Germany

\begin{abstract}
In the calculation of eigenfrequencies of 3-D metallic nanostructures occurs the challenge that the material parameters depend on the desired eigenfrequency. We propose a formulation where this leads to a polynomial eigenvalue problem which can be tackled by different solving strategies. A comparison between a Newton-type method and a Jacobi-Davidson algorithm is given.
\end{abstract}

\section{Introduction}

The focus of our analysis is on nanostructures which include a metallic substructure. In the microwave spectrum metals can often be treated as perfect conductors without a significant loss of accuracy. If, however, the nanostructures are supposed to operate at optical frequencies, the finite conductivity of the metallic parts and their frequency dependence must not be neglected. Since we are interested in the computation of eigensolutions of such nanostructures, there is the challenge that the operating frequency (the eigenvalue) is not a-priori known. Thus, the material dispersion leads to a nonlinear eigenvalue formulation.

The rest of the paper is organized as follows: Sect. 2 briefly reviews the material behavior of metals at optical frequencies. In Sect. 3 we derive in the first part a continuous eigenvalue representation which is able to take the dispersion into account. In the second part of Sect. 3 this representation is discretized. Section 4 reviews several solving strategies for polynomial eigenvalue problems. Finally a numerical example is presented in Sect. 5.

\section{Metals at optical frequencies}

There are several publications which investigate experimentally the optical properties of noble metals such as gold and silver (Johnson and Christy, 1972; Ordal et al., 1983; Palik,

Correspondence to: $\mathrm{B}$. Bandlow (bandlow@tet.upb.de)
1997; Rakic et al., 1998). It turns out that the material values obtained by measurements can be fairly approximated by Drude or a Drude-Lorentz models. These are rational functions which are able to depict one or more resonance effects in a specific frequency range. In general the dependency of the permittivity on the frequency $\epsilon(\omega)$ shall be approximated by a general 2 nd order model

$\epsilon(\omega)=\epsilon_{0}\left(\epsilon_{\infty}+\frac{\beta_{0}+j \omega \beta_{1}}{\alpha_{0}+j \omega \alpha_{1}-\omega^{2}}\right)$.

Here $\epsilon_{0}$ is the permittivity of free-space, the parameters $\epsilon_{\infty}$, $\alpha_{0}, \alpha_{1}, \beta_{0}$ and $\beta_{1}$ are real-valued, and an $e^{j \omega t}$ time dependency is implied. Figure 1 exemplarily shows the real and imaginary part of the relative permittivity of silver in the infrared range. The data from (Johnson and Christy, 1972) can be fitted by the parameter set $\epsilon_{\infty}=5, \alpha_{0}=0, \alpha_{1}=3.22 e 13$, $\beta_{0}=1.96 e 32, \beta_{1}=0$ of a Drude approximation.

The imaginary part of the complex permittivity can be reinterpreted as a conductivity, and from the conductivity the skin-depth can easily be calculated. For instance, at a wavelength of 1.5 microns we obtain a skin-depth of $140 \mathrm{~nm}$, which implies that we can not model thin sheets of a material like silver as a good or even perfect electrical conductor at optical frequencies.

\section{Eigenmode formulation}

\subsection{Non-linear and polynomial eigenvalue problem}

In the following, we derive the non-linear eigenvalue formulation for structures with dispersive materials. For the sake of simplicity, we assume a homogeneous medium and begin in the continuous rather than the discrete regime. The eigenmode formulation for the electrical field strength $\boldsymbol{E}$ follows from Maxwell's equations in frequency domain and reads

$\operatorname{curl} \frac{1}{\mu} \operatorname{curl} \boldsymbol{E}=\omega^{2} \epsilon(\omega) \boldsymbol{E}$. 


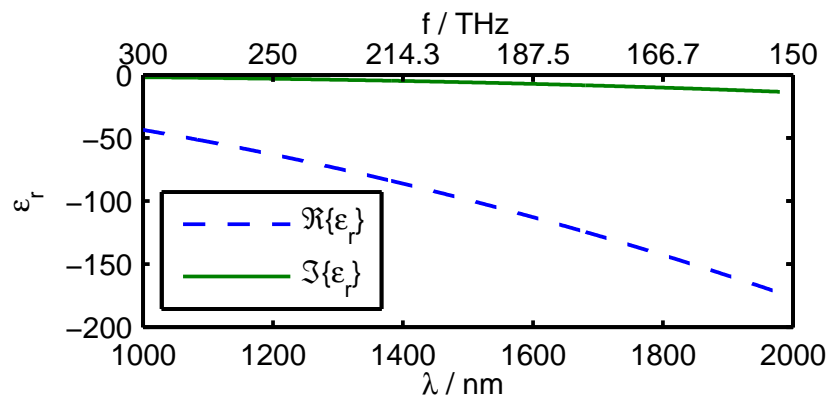

Fig. 1. Real and imaginary part of the relative permittivity of silver in the infrared range.

The dispersion of the permittivity $\epsilon(\omega)$ shall be given by a Drude model as motivated in Sect. 2:

$\epsilon(\omega)=\epsilon_{0}\left(\epsilon_{\infty}+\frac{\beta_{0}}{j \omega \alpha_{1}-\omega^{2}}\right)$.

We insert Eq. (3) into Eq. (2) and obtain the complex, nonhermitian, polynomial eigenvalue problem (PEP) in $\omega$

$\left(\omega^{3} a_{3}+\omega^{2} a_{2}+\omega a_{1}+a_{0}\right) \boldsymbol{E}=0$.

The coefficients $a_{i}$ are given by

$a_{0}=-j \alpha_{1} A_{c c}, \quad a_{1}=\epsilon_{0} \beta_{0}+A_{c c}$,

$a_{2}=j \epsilon_{0} \epsilon_{\infty} \alpha_{1}, \quad a_{3}=-\epsilon_{0} \epsilon_{\infty}, \quad A_{c c}=\operatorname{curl} \frac{1}{\mu}$ curl.

\subsection{Discrete formulation}

The eigenvalue formulation is discretized using the finite integration technique (FIT) (Weiland, 1977, 1996). In this very general framework Maxwell's equations are transformed into algebraic equations - the Maxwell's Grid Equations - which can be used further to formulate e.g. a discrete wave equation. The degrees of freedom of the FIT approach are the so-called grid voltages $\widehat{\boldsymbol{e}}$, which are defined on the edges of a three-dimensional Cartesian grid. Finally, the discrete representation of the non-linear eigenvalue problem Eq. (2) reads

$\mathbf{A}_{c c} \widehat{\boldsymbol{e}}=\omega^{2} \mathbf{M}_{\varepsilon}(\omega) \widehat{\boldsymbol{e}}$.

Here, the large and sparse matrix $\mathbf{A}_{c c}$ is the curl-curl system operator and includes the double curl operation as well as the permeability distribution of the structure. The diagonal matrix $\mathbf{M}_{\varepsilon}$ is the generalized permittivity operator. The searched eigenvalue is the squared angular frequency $\omega^{2}$, and the field distribution is defined by the eigenvector $\widehat{\boldsymbol{e}}$. The dimension of the problem is $N_{e} \times N_{e}$, with $N_{e}$ the number of grid edges.

In a straight forward manner, we can also find a discrete representation of the PEP Eq. (4) using FIT

$\Psi(\omega) \widehat{\boldsymbol{e}}=\left(\omega^{3} \mathbf{A}_{3}+\omega^{2} \mathbf{A}_{2}+\omega \mathbf{A}_{1}+\mathbf{A}_{0}\right) \widehat{\boldsymbol{e}}=0$, where $\mathbf{A}_{i}$ are the spatially discretized coefficient matrices corresponding to the $a_{i}$ of Eq. (4). This representation also supports an arbitrary inhomogeneous material distribution (including the dispersive permittivity), and it turns out that the usual facet-weighted averaging procedure at material interfaces does not need any special treatment. In FIT with Cartesian grids the matrices $\mathbf{A}_{3}$ and $\mathbf{A}_{2}$ are diagonal, and $\mathbf{A}_{1}$ and $\mathbf{A}_{0}$ are sparse. The resulting PEP is complex and nonHermitian.

\section{Solver for polynomial eigenvalue problems}

The PEP from Sect. 3.2 can be solved in different ways, and we briefly discuss four variants.

\subsection{Fixed-point iteration}

A first idea is to evaluate $\mathbf{M}_{\varepsilon}(\omega)$ at a certain frequency $\omega_{i}$ and to solve a standard linear eigenvalue problem from Eq. (5) for the eigenfrequency $\omega_{i+1}$ :

$\mathbf{A}_{c c} \widehat{\boldsymbol{e}}=\omega_{i+1}^{2} \mathbf{M}_{\varepsilon}\left(\omega_{i}\right) \widehat{\boldsymbol{e}}$.

This approach defines a fixed-point iteration process $\omega_{i+1}=\Phi\left(\omega_{i}\right)$, where the operator $\Phi$ includes a solving step of standard linear eigenvalue problem.

This scheme works well (but not very fast) in many cases. However, the proof of its convergence for general cases e.g. using Banach's fixed-point theorem - is quite challenging due to the complex nature of the eigenvalue problem involved.

\subsection{Linearization via companion matrix}

A direct way to solve any PEP is to use the so-called companion matrix of the PEP. A PEP of order $i$ can be recast into a generalized linear eigenvalue problem of the form $\mathbf{A x}=\lambda \mathbf{B x}$, where

$\mathbf{A}=\left[\begin{array}{cccc}0 & \mathbf{I} & \cdots & 0 \\ \vdots & \vdots & \ddots & \vdots \\ \vdots & \vdots & \vdots & \mathbf{I} \\ -\mathbf{A}_{0} & -\mathbf{A}_{1} & \cdots & -\mathbf{A}_{i-1}\end{array}\right]$,

$\mathbf{B}=\left[\begin{array}{llll}\mathbf{I} & & & \\ & \ddots & & \\ & & \mathbf{I} & \\ & & & \mathbf{A}_{i}\end{array}\right], \quad \mathbf{x}=\left[\begin{array}{c}\widehat{\boldsymbol{e}} \\ \omega \widehat{\boldsymbol{e}} \\ \vdots \\ \omega^{i-1} \widehat{e}\end{array}\right]$.

For a problem of polynomial order $i$ with $n \times n$ matrices, this approach leads to $(i-1) \times n$ additional eigenvalues, which are not necessarily all solutions of the original PEP and therefore have to be dropped. Since this approach leads to large and typically ill-conditioned matrices, it is only feasible for small PEPs. 


\subsection{Newton-type methods for PEPs}

The Newton method for polynomial eigenvalue problems may be regarded as a generalization of the method of inverse iteration (Schreiber, 2008). The algorithm yields one eigenpair at a time and is based on a function $f$ of the eigenvector $\boldsymbol{u}$ and the eigenvalue $\omega$

$f\left(\left[\begin{array}{l}\boldsymbol{u} \\ \omega\end{array}\right]\right)=\left(\left[\begin{array}{c}\Psi(\omega) \boldsymbol{u} \\ \boldsymbol{w}^{H} \boldsymbol{u}-1\end{array}\right]\right)$.

It includes the matrix polynomial $\Psi(\omega)$ from Eq. (6) and a normalization vector $\boldsymbol{w}$, which has to be chosen such that $\boldsymbol{w}^{H} \boldsymbol{u}=1$ holds throughout the iteration. The first derivative of Eq. (9) is given by the Jacobian

$J\left(\left[\begin{array}{l}\boldsymbol{u} \\ \omega\end{array}\right]\right)=\left[\begin{array}{cc}\Psi(\omega) & \Psi^{\prime}(\omega) \boldsymbol{u} \\ \boldsymbol{w}^{H} & 0\end{array}\right]$

where $\Psi^{\prime}(\omega)$ denotes the derivation of Eq. (6). For a given starting guess of the eigenpair $\left(\boldsymbol{u}_{0}, \omega_{0}\right)$, the Newton correction at step $i$ is defined by

$J\left(\left[\begin{array}{l}\boldsymbol{u}_{i} \\ \omega_{i}\end{array}\right]\right)\left[\begin{array}{l}\Delta \boldsymbol{u}_{i+1} \\ \Delta \omega_{i+1}\end{array}\right]=-f\left(\left[\begin{array}{l}\boldsymbol{u}_{i} \\ \omega_{i}\end{array}\right]\right)$

and

$\boldsymbol{u}_{i+1}=\boldsymbol{u}_{i}+\Delta \boldsymbol{u}_{i+1}, \quad \omega_{i+1}=\omega_{i}+\Delta \omega_{i+1}$.

Therefore, the linear system Eq. (11) has to be solved in each iteration step. The Newton algorithm is terminated if the norm of the residual

$\boldsymbol{r}=\Psi(\omega) \boldsymbol{u}$

is sufficiently small.

\subsection{Jacobi-Davidson algorithm for PEPs}

The PEP in the form Eq. (6) can also be solved using a Jacobi-Davidson algorithm (JD) (Sleijpen et al., 1996; Bai et al., 2000). This method is intended to find one or more interior eigenvalues of the spectrum near a given target $\omega_{0}$.

The main idea within the JD method is to project the PEP on a low-dimensional orthogonal subspace $\mathbf{V}$, which leads to a low-dimensional PEP with coefficient matrices $\mathbf{M}_{i}=\mathbf{V}^{*} \mathbf{A}_{i} \mathbf{V}$. The low-dimensional PEP can be solved by the companion matrix approach from Sect. 4.2 and any method for generalized eigenvalue problems. The low-dimensional eigenvector $\boldsymbol{s}$ is expanded to full size again, $\boldsymbol{u}=\mathbf{V} \boldsymbol{s}$, which leads to the current approximative eigenvector $\boldsymbol{u}$. Since $\boldsymbol{u}$ is an approximation, the residual Eq. (12) has to be calculated, and the process stops if the norm of $\boldsymbol{r}$ is sufficiently small. Since this typically does not occur after the first iteration step, a so-called correction equation is formulated and solved, which produces an additional vector, which extends the subspace.
This procedure is repeated until convergence. The computationally most expensive task inside the JD iteration is the solution of the correction equation, which reads

$$
\left(\mathbf{I}-\frac{p \boldsymbol{u}^{*}}{\boldsymbol{u}^{*} \boldsymbol{p}}\right) \Psi(\theta)\left(\mathbf{I}-\boldsymbol{u} \boldsymbol{u}^{*}\right) \boldsymbol{t}=-\boldsymbol{r} .
$$

Here, $\Psi$ is the polynomial from Eq. (6) evaluated at the last, best estimation $\theta$ (namely the Ritz value), $t$ is the correction vector, $\boldsymbol{r}$ the residual, $\boldsymbol{u}=\mathbf{V} \boldsymbol{s}$ the best actual approximation of the searched eigenvector, and $p=\Psi^{\prime}(\theta)$. In the beginning of the JD process the user-defined target $\omega_{0}$ may be more accurate than an extracted Ritz value. Therefore we set $\boldsymbol{p}=\Psi^{\prime}\left(\omega_{0}\right)$ until the residual is below a predefined tolerance, in order to get a better correction vector $\boldsymbol{t}$. (This was proposed in (Hochstenbach and Sleijpen, 2008).) For the solution of the correction Eq. (13) we use a preconditioned bicgstab(1) method (Sleijpen and Fokkema, 1993). As preconditioner we use an LU decomposition of the polynomial evaluated at the target value $\omega_{0}$. Therefore the LU decomposition has to be established only once per JD run.

\subsection{Validation}

A simple way to validate a specific implementation from the Newton method from Sect. 4.3 or the JD method from Sect. 4.4 is to go back to the fixed-point formulation in Eq. (7) and to execute one single step of the iteration. In all numerical tests our results yield accuracies in the range of numerical noise.

\section{Numerical example}

As an example we take a bow tie slot antenna which can be regarded as a resonator at optical wavelengths. A potential application is in spectroscopy where field confinement and focusing is desirable. An extensive study can be found e.g. in Guo et al., 2008.

\subsection{Setup}

The structure consists of a layer of silver and the dimensions are given in Fig. 2. The material parameters of silver are taken from Sect. 2, where Eq. (1) returns a Drude model Eq. (3) and the resulting PEP is of order three. The permeability is chosen to be $\mu=\mu_{0}$. For the geometric modeling we use the commercial tool CST MicrowaVE STUDIO (Computer Simulation Technology AG (CST)). The discretization of one quarter of the whole structure (due to symmetry) uses a rather coarse mesh with only $15 \times 16 \times 22$ grid lines, leading to 13657 complex degrees of freedom. (Note that in this presentation we concentrate on solving strategies rather than the convergence of our grid model towards the "real" solution.) In order to model a whole array of bow tie 

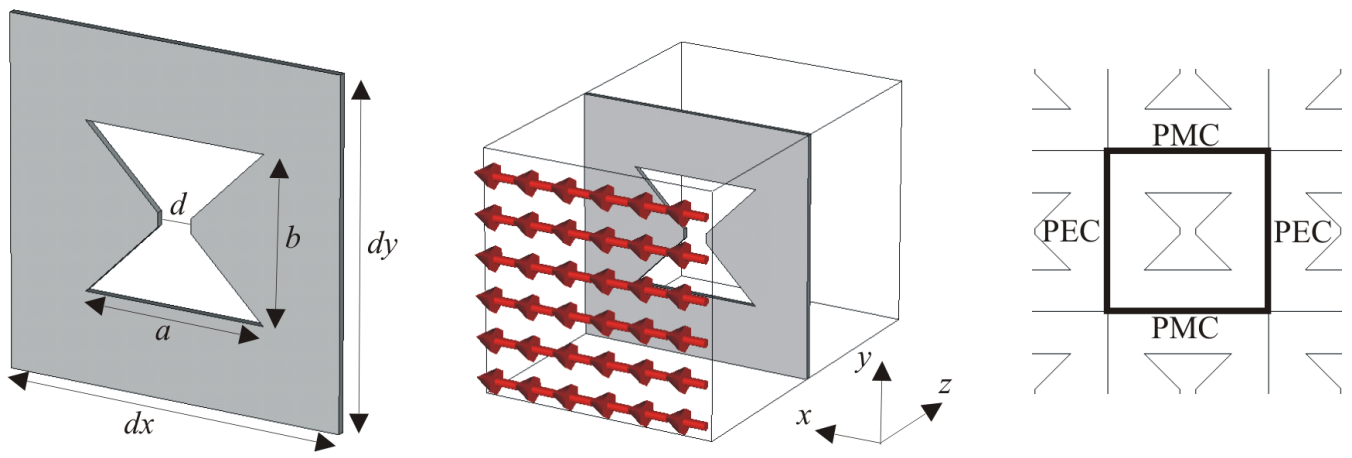

Fig. 2. Bow tie antenna structure with $a=270 \mathrm{~nm}, b=240 \mathrm{~nm}, d=50 \mathrm{~nm}$ and thickness of $t=10 \mathrm{~nm}$. The transversal dimensions are $d x=d y=500 \mathrm{~nm}$. The polarization of the incident TEM wave and the resulting transversal boundary conditions are shown at the center and at the right.

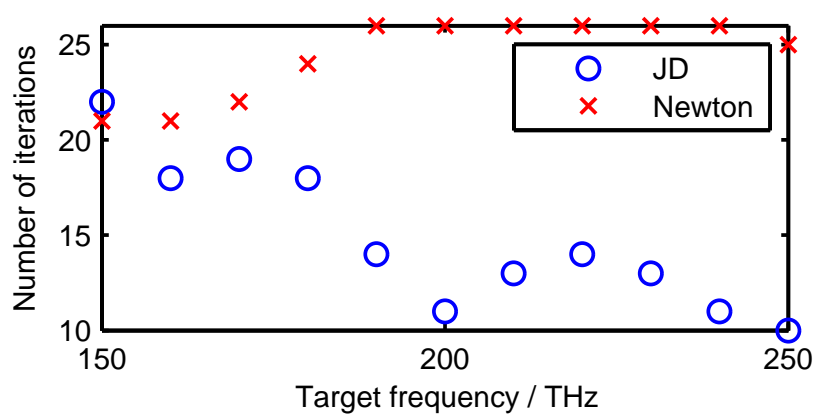

Fig. 3. Number of iterations of the JD and Newton method to converge for different target frequencies $\omega_{0}$.

antennas illuminated by a plane wave, the transversal boundary conditions are chosen to be perfectly electric and perfectly magnetic conducting (cf. Fig. 2). In propagation direction we apply Berenger's perfectly matched layer (PML) (Berenger, 1994) to truncate the computational domain appropriately. The PML itself consists of frequency dependent materials, but here we evaluate the PML once at our starting frequency $\omega_{0}$ instead of including its frequency dependence into the eigenvalue formulation. Nevertheless, to determine the eigenfrequency of the bow tie antenna, a complex non-Hermitian polynomial eigenvalue problem has to be solved, and we compare the results of the Newton method from Sect. 4.3 to those of the JD method from Sect. 4.4, both of them implemented in MATLAB. Convergence is supposed to be reached when the norm of the residual Eq. (12) is less than 1e-9.

\subsection{Results}

An estimation of the eigenfrequency can be obtained, e.g., by measurements or a scattering parameter simulation, see

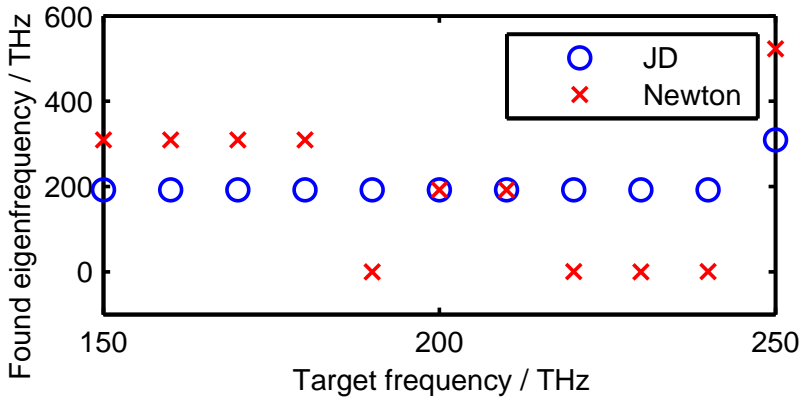

Fig. 4. Resulting eigenvalues of the JD and Newton method for different target frequencies $\omega_{0}$.

(Guo et al., 2008) for details. In our case the searched eigenfrequency is at $192.4 \mathrm{THz}$.

Figure 3 shows the number of iterations needed by the Jacobi-Davidson and Newton method to generate an eigenpair for different target frequencies $\omega_{0}$. In almost all cases the Jacobi-Davidson method needs less iterations than the Newton method for our specific setup. However, it is questionable which eigenpairs are actually found

Figure 4 shows the eigenfrequencies generated by the Jacobi-Davidson and Newton method for different target frequencies $\omega_{0}$. It turns out that the result of the Newton method strongly depends on the chosen target frequency $\omega_{0}$ for the first step of Eq. (11). On the contrary the Jacobi-Davidson method reliably generates the same eigenvalue also for less accurately chosen target frequencies.

Figure 5 shows the time needed for the computation of the eigenfrequency (including only those runs which led to the desired eigenvalue of $192.4 \mathrm{THz}$ ). Again the JD method outperforms the Newton approach in all cases considered here.

Finally Fig. 6 shows the distribution of electrical field strength of the eigenfrequency at $192.4 \mathrm{THz}$. It is mainly 


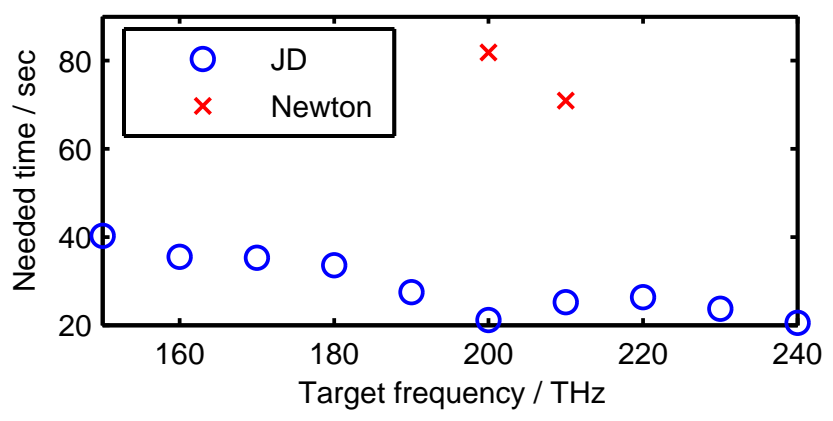

Fig. 5. Computation times of JD and Newton method for target frequencies, which lead to the searched eigenvalue.

orientated in $x$-direction (like the incident plane wave) and is concentrated within the slot of the bow tie antenna as proposed in the literature.

\section{Conclusions}

After a review of the behavior of metals at optical frequencies, an eigenfrequency formulation which is capable to deal with frequency dispersive materials has been presented. The formulation is based on the finite integration technique and leads to a polynomial eigenvalue problems (PEP). Four strategies to solve this PEP have been presented and tested. As a numerical example we have used a bow tie slot antenna (Guo et al., 2008) which consists of silver and operates at a wavelengths around 1.5 microns. We have computed its resonance frequency and compared the Newton method and the Jacobi-Davidson method. It turns out that the JacobiDavidson method is more robust in terms of poorly chosen target frequencies and also faster at least in our implementation.

If the match of measurement data of material properties requires higher order polynomial functions, our formulation of Sect. 3 can easily be extended and will lead to higher order matrix polynomials.

\section{References}

Bai, Z., Demmel, J., Dongarra, J., Ruhe, A., and van der Vorst, H.: Templates for the Solution of Algebraic Eigenvalue Problems: A Practical Guide, SIAM, Philadelphia, 2000.

Berenger, J.-P.: A perfectly matched layer for the absorption of electromagnetic waves, J. Comput. Phys., 114, 185-200, 1994.

Computer Simulation Technology AG (CST): CST Studio Suite 2008, http://www.cst.com, 2008.

Guo, H., Meyrath, T. P., Zentgraf, T., Liu, N., Fu, L., Schweizer, H., and Giessen, H.: Optical resonances of bowtie slot antennas and their geometry and material dependence, Opt. Express, 16, 7756-7766, http://www.opticsexpress.org/abstract. cfm?URI=oe-16-11-7756, 2008.

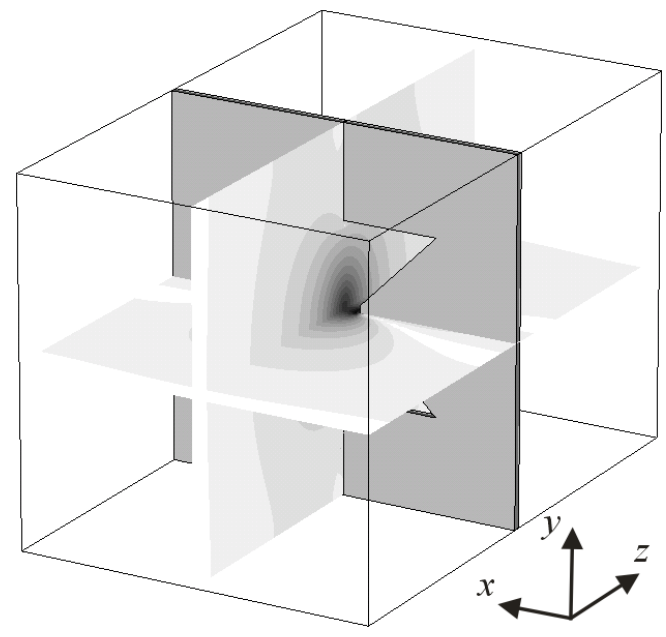

Fig. 6. Contour plot of the field distribution of the eigensolution at $192.4 \mathrm{THz}$ : The $x$-component of the electrical field strength is confined within the slot of the bow tie antenna.

Hochstenbach, M. E. and Sleijpen, G. L. G.: Harmonic and refined Rayleigh-Ritz for the polynomial eigenvalue problem, Numerical Linear Algebra with Applications, 15, 35-54, 2008.

Johnson, P. B. and Christy, R. W.: Optical Constants of the Noble Metals, Phys. Rev. B., 6, 4370-4379, 1972.

Ordal, M. A., Long, L. L., Bell, R. J., Bell, S. E., Bell, R. R., R. W. Alexander, J., and Ward, C. A.: Optical properties of the metals $\mathrm{Al}, \mathrm{Co}, \mathrm{Cu}, \mathrm{Au}, \mathrm{Fe}, \mathrm{Pb}, \mathrm{Ni}, \mathrm{Pd}, \mathrm{Pt}, \mathrm{Ag}, \mathrm{Ti}$, and $\mathrm{W}$ in the infrared and far infrared, Appl. Opt., 22, 1099-1119, http://ao.osa.org/abstract.cfm?URI=ao-22-7-1099, 1983.

Palik, E. D.: Handbook of Optical Constants of Solids , 1997.

Rakic, A. D., Djurišic, A. B., Elazar, J. M., and Majewski, M. L.: Optical Properties of Metallic Films for Vertical-Cavity Optoelectronic Devices, Appl. Opt., 37, 5271-5283, http://ao.osa.org/ abstract.cfm?URI=ao-37-22-5271, 1998.

Schreiber, K.: Nonlinear Eigenvalue Problems: Newton-type Methods and Nonlinear Rayleigh Functionals, Ph.D. thesis, TU Berlin, http://nbn-resolving.de/urn:nbn:de:kobv:83-opus-18754, 2008.

Sleijpen, G. L. G. and Fokkema, D. R.: Bi-CGSTAB(1) for linear equations involving unsymmetric matrices with complex spectrum, Elec. Trans. Numer. Anal., 1, 11-32, 1993.

Sleijpen, G. L. G., Booten, A. G. L., Fokkema, D. R., and der Vorst, H. A. V.: Jacobi-Davidson type methods for generalized and polynomial eigenproblems, BIT, 36, 595-633, 1996.

Weiland, T.: Eine Methode zur Lösung der Maxwellschen Gleichungen für sechskomponentige Felder auf diskreter Basis, Archiv für Elektronik und Übertragungstechnik, 31, 116-120, 1977.

Weiland, T.: Time Domain Electromagnetic Field Computation with Finite Difference Methods, International Journal of Numerical Modelling, 9, 295-319, 1996. 